\title{
Silicon meet graphene for a new family of near- infrared Schottky photodetectors
}

\author{
Maurizio Casalino \\ Institute for Microelectronics and Microsystems - Italian National Council of Research \\ Correspondence: maurizio.casalino@na.imm.cnr.it; Tel.: +39-081-6132345
}

\begin{abstract}
In recent years graphene has attracted much interest due to its unique properties of flexibility, strong light-matter interaction, high carrier mobility and broadband absorption. In addition, graphene can be deposited on many substrates including silicon with which is able to form Schottky junctions opening the path to the realization of near-infrared silicon photodetectors based on the internal photoemission effect where graphene play the role of the metal. In this work, we review the very recent progress of the near-infrared photodetectors based on Schottky junctions involving graphene. This new family of device promises to overcome the limitations of the Schottky photodetectors based on metals showing the potentialities to compare favorably with germanium photodetectors currently employed in silicon photonics.
\end{abstract}

Keywords: silicon; graphene; internal photoemission; field-effect transistor; photodetector; nearinfrared; waveguide

\section{Introduction}

Silicon photonics is nowadays an emerging market promising to reach a value of $\$ 560 \mathrm{M}$ at chip level and $\$ 4 \mathrm{~B}$ at transceiver level in the 2025. Indeed, both switching and interconnects of the existing data center risk becoming early a bottleneck for the huge increase in internet data traffic driven by the social network and video contents.

Silicon photonics transceivers market forecast

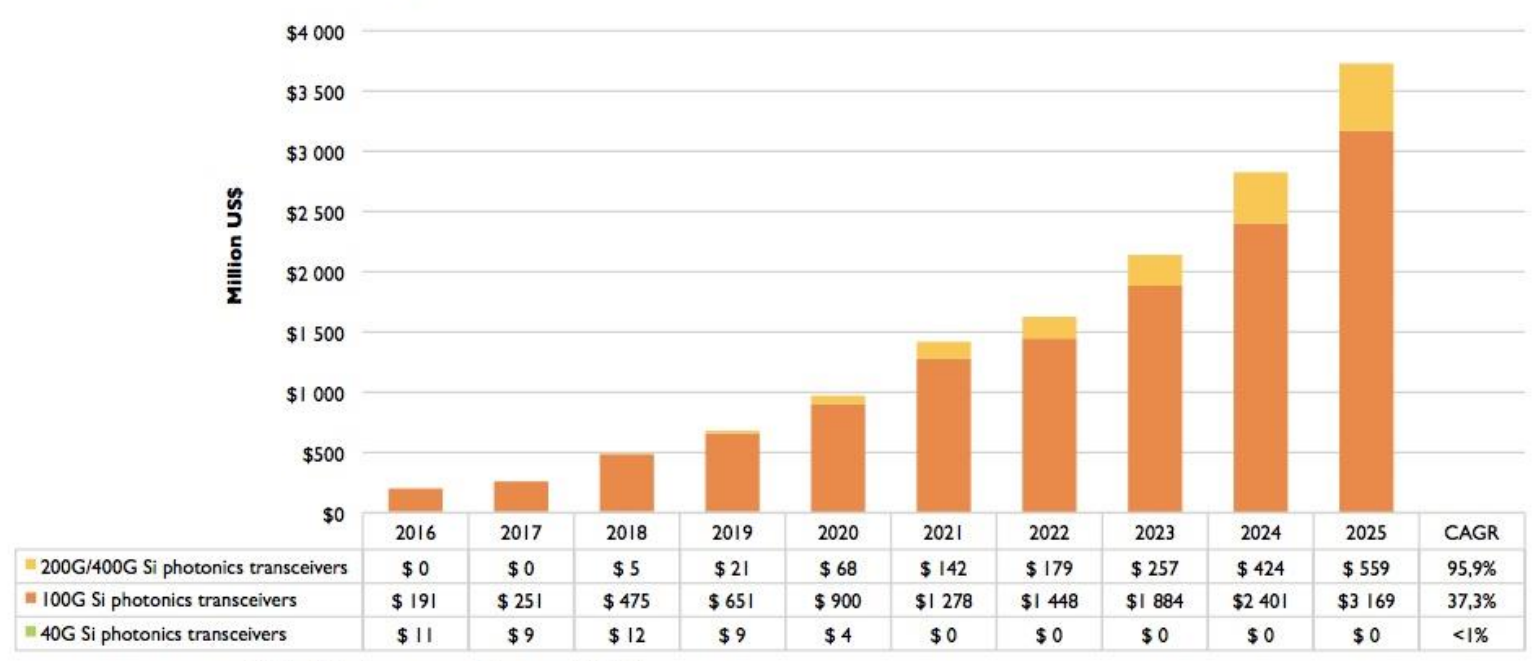

(Yole Développement, January 2018)

Figure 1. Silicon photonics 2013-2025 market forecast [1].

This is because in the next future new technologies must necessarily to be introduced. The research activity in Silicon photonics can be dated back to the 1980s and in the last years more and more players are going in this industry's landscape. Nowadays Intel shares with Luxtera the 
leadership in this field with products for 100G data rates already present on the market. Because Silicon is a mature technology developed in the years of the strong expansion of the microelectronic industry, the use of $\mathrm{Si}$ for the fabrications of photonic components meet the requirements of the data center in term of low cost, reliability, integration density and power consumption. A key component in Si photonics is constituted by the photodetectors (PDs), devices that are able to convert the incoming light into current.

Si PDs are commercial devices in the visible spectrum, however, the employment of Si for the realization of PD operating at near-infrared (NIR) wavelengths is not a simple task due to $\mathrm{Si}$ transparence at wavelengths above $1.1 \mu \mathrm{m}$. NIR Si-based PDs take conventionally advantage of germanium $(\mathrm{Ge})$ integration $[2,3]$ on $\mathrm{Si}$, however, due to a $4.3 \%$ lattice mismatch very high leakage current hinders the device performance. This issue can be mitigated by realizing a buffer layer based on a two-step epitaxial growth technique $[4,5]$ that minimize but, unfortunately, do not remove the defect center responsible of the high leakage current. Moreover this buffer layer causes problems in both planarity and thermal budget [6] preventing the possibility of a monolithic Ge integration on Si. In addition Ge is characterized by a low absorption at $1550 \mathrm{~nm}$ with respect to gallium arsenide (InGaAs) leading to thick active intrinsic region of the PIN structures and so slow devices.

In this context an all-Si approach is preferred and one option it is offered by the internal photoemission effect (IPE). IPE occurs in a metal/semiconductor Schottky junction when due to an incident optical radiation the carriers of the metal are excited to energies above the Schottky barrier of the junction and transported in the conduction band of the semiconductor [7,8]. IPE has been widely used in high-performance platinum silicide ( $\mathrm{PtSi})$ and palladium silicide $\left(\mathrm{Pd}_{2} \mathrm{Si}\right)$ Schottky barrier infrared CCD image sensors. The first generation of detectors was based on a Pd2Si/Si Schottky junctions and was developed for operating in the spectrum from 1 to $2.4 \mu \mathrm{m}$ for satellite applications and it required an operating temperature of $120 \mathrm{~K}[9,10]$. Subsequently PtSi/Si Shottky junctions showed the capability to work at longer wavelengths, from 3 to $5 \mu \mathrm{m}[11,12]$. The $512 \times 512$ focal plane array based on PtSi require operating temperature of $80 \mathrm{~K}$ [13]. In these devices due to the low Schottky Barrier Height (SBH) the cryogenic temperature were necessary to minimize the noise current.

In the 2006, it was proposed to work with higher SBH in order to use IPE also at room temperature and to recovery the consequently reduced efficiency by means of Fabry-Peròt resonant structures [14]. Subsequently, not only these devices were realized [15] but many other strategies were employed in order to increase the efficiency of IPE-based Si PDs at room temperature. Indeed, IPE was investigated in metallic structures at nanoscale as: Si nanoparticles (NPs) [16], stripes able to support surface plasmon polaritons (SPPs) [17, 18], gratings [19] and antennas [20]. In any case, by using metal/silicon junctions, responsivities of only $5 \mathrm{~mA} / \mathrm{W}$ [21] and $30 \mathrm{~mA} / \mathrm{W}$ [16] were reported for vertically-illuminated and waveguide PDs, respectively. This low responsivity is mainly due to the fact that the carriers photo-excited in the metal have a low probability to be emitted over the Schottky barrier. However it is well-known that the carrier emission probability can be increased by reducing the metal thickness, or, in other words, that the lower the metal thickness the higher the emission probability [22,23]. This consideration leads to the idea of replacing the metals with 2D materials and, in this context, graphene is of course a good candidate. Si-based graphene PDs are typically based on field effect transistor (FET) structures and many review articles are reported about them in literature [24,25]. However, graphene PDs based on FET structures are characterized by some drawbacks: a low photo-conversion area (low efficiency) due to the fact that the internal electric field is formed only in narrow region $(200 \mathrm{~nm})$ close to the source and drain edges [26] and a further high dark current generated in any case when a bias drops between source and drain. On the contrary IPEbased graphene/Si PDs promises to overcome these limitations because the photo-conversion area is linked to the graphene area in contact with $\mathrm{Si}$ and because the dark current is the saturation current of a Schottky diode reversely biased.

In this review article the emerging field of the NIR IPE-based graphene/Si PDs, is presented. In the first section, IPE theory for junctions involving 2D materials will be elucidated and put in comparison with the classical theory used for metals. Then, the main both vertically illuminated and 
waveguide NIR IPE-based graphene/Si PDs will be reviewed and discussed, comparing the performance of structures based on the different approaches. Finally, will be highlighted as these PDs have the potentialities to compare favorably with the Ge technology in the field of NIR detection, also offering new advantageous characteristics.

\section{Theoretical background}

In order to quantify the performance of IPE-based PDs, a very important parameter is the internal quantum efficiency $\eta_{\text {int, }}$ i.e., the numbers of carriers collected per absorbed photons. On the other hands, the external quantum responsivity $\eta_{\text {ext }}$ (number of carriers collected per incident photons) is linked to the internal by $\eta_{\mathrm{ext}}=\mathrm{A} \eta_{\mathrm{int}}$, being $\mathrm{A}$ the active material absorption. A macroscopic magnitude is the responsivity $R$, i.e., the ratio between the photogenerated current $\left(\mathrm{I}_{\mathrm{ph}}\right)$ and the incident optical power (Pinc). The responsivity $R$ is linked to the external quantum efficiency $\eta_{\text {ext }}$ by the following:

$$
R=\frac{I_{p h}}{P_{i n c}}=\frac{[\mathrm{nm}]}{1242} \times_{e x t}
$$

In the 1931 Fowler derived the first model of carrier photoemission from metal into vacuum [27]. In the 1960s, Cohen, Vims and Archer [28] modified the Fowler's theory to take into account the carrier photoemission into a semiconductor. Subsequently, Elabd and Kosonocky reviewed the previous model and, under the zero temperature approximation, they obtained that the device internal quantum efficiency $\eta^{3 \mathrm{D}_{\text {int }}}$ (number of carriers collected per absorbed photons) can be written as [22]:

$$
{ }_{\text {int }}^{3 D}=\frac{1}{8 q_{B 0}} \times \frac{\left(\begin{array}{ll}
h & q_{B 0}
\end{array}\right)^{2}}{h}
$$

where the factor $1 / 8 q \Phi_{B 0}{ }^{35}$ is very often replaced by a generic factor $C$ (named quantum efficiency coefficient) used to put in agreement the theory with the experimental data, $h v=1242 / \lambda_{0}(\mathrm{~nm})$ is the photon energy, $\lambda_{0}$ is the vacuum wavelength, $\Phi_{B 0}$ is the Schottky barrier height (SBH) under zero bias, and q is the electron charge. The 3D apex is used to indicate that the formula refers to junctions involving 3D materials, i.e., metals.

It is worth noting that Eq. 2 was derived for junctions based on metals, i.e., on three-dimensional materials, however, it has been proved that it fails to correctly describe carrier photoemission involving Schottky junctions based on two-dimensional materials [29,30], thus IPE theory must be properly modified in order to be applied to the Graphene/Si junctions whose band diagram is reported in Fig. 2.

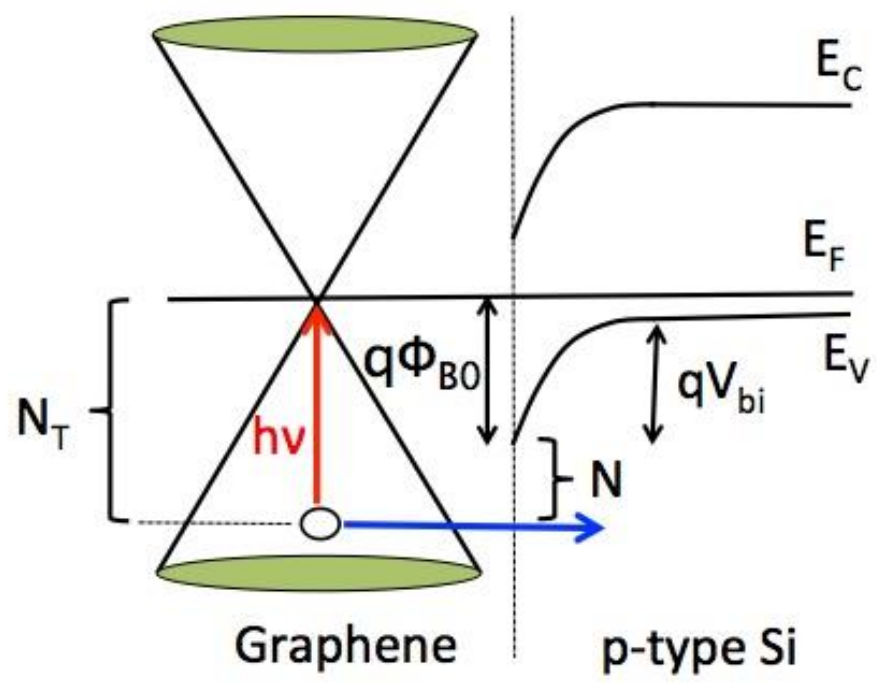


Figure 2. Band diagram of a graphene/p-Si Schottky junction: EF is the Fermi energy of the metal, EV is the Si valence band energy, EC is the Si conduction band energy, qФB is the Schottky barrier height and qVbi is the built-in energy. NT and N are the total number of possible excited hole states and the number of states from which hole emission into Si can occur across the barrier height of qФB0, respectively. by:

By following Elabd's approach [22] the total number of possible excited states for holes is given

$$
N_{T}={ }_{0}^{h} D(E) \times d E
$$

where $\mathrm{D}(\mathrm{E})$ is the density of states (DOS) of graphene that can be written as $D(E)=\frac{2 E}{\pi \hbar^{2} v_{F}^{2}}[31], E$ is the hole energy with reference to the Fermi level, $v_{F}$ is the Fermi velocity, $\hbar$ is the reduced Planck constant, and $h v$ is the photon energy. In addition, the number of states $N$ from which the hole can be emitted over the $\operatorname{SBH} q \Phi_{B 0}$, is:

$$
N={ }_{q}^{h} D(E) \times P(E) \times d E
$$

where $P(E)$ is the carrier emission probability.

In three-dimensional materials, $P(E)$ can be expressed as $P(E)=(1-\cos \theta) / 2$ [22], where $\theta$ is the so defined carrier escape angle [22]. On the contrary, $P(E)$ in graphene assumes a very simple value of $1 / 2$ because the $\pi$ orbitals are always normal to the graphene/Si interface, thus the photo-excited hole momenta can have two directions: one pointing towards Si and the other in the opposite direction [29]. Therefore, the graphene internal quantum efficiency $\eta^{2 D_{\text {int }}}$ can be derived as $[29,30]$ :

$$
{ }_{\text {int }}^{2 D}=\frac{N}{N_{T}}=\frac{1}{2} \times \frac{(h)^{2}\left(q_{B 0}\right)^{2}}{(h)^{2}}
$$

The $2 \mathrm{D}$ apex is used to indicate that the formula refers to junctions involving $3 \mathrm{D}$ materials, i.e., graphene.

In Fig. 3 is reported the internal quantum efficiency versus the SBH for three different wavelengths of $0.85 \mu \mathrm{m} .1 .3 \mu \mathrm{m}$ and $1.55 \mu \mathrm{m}$ in order to show that in any case the IPE effect is enhanced in junctions involving 2D materials.

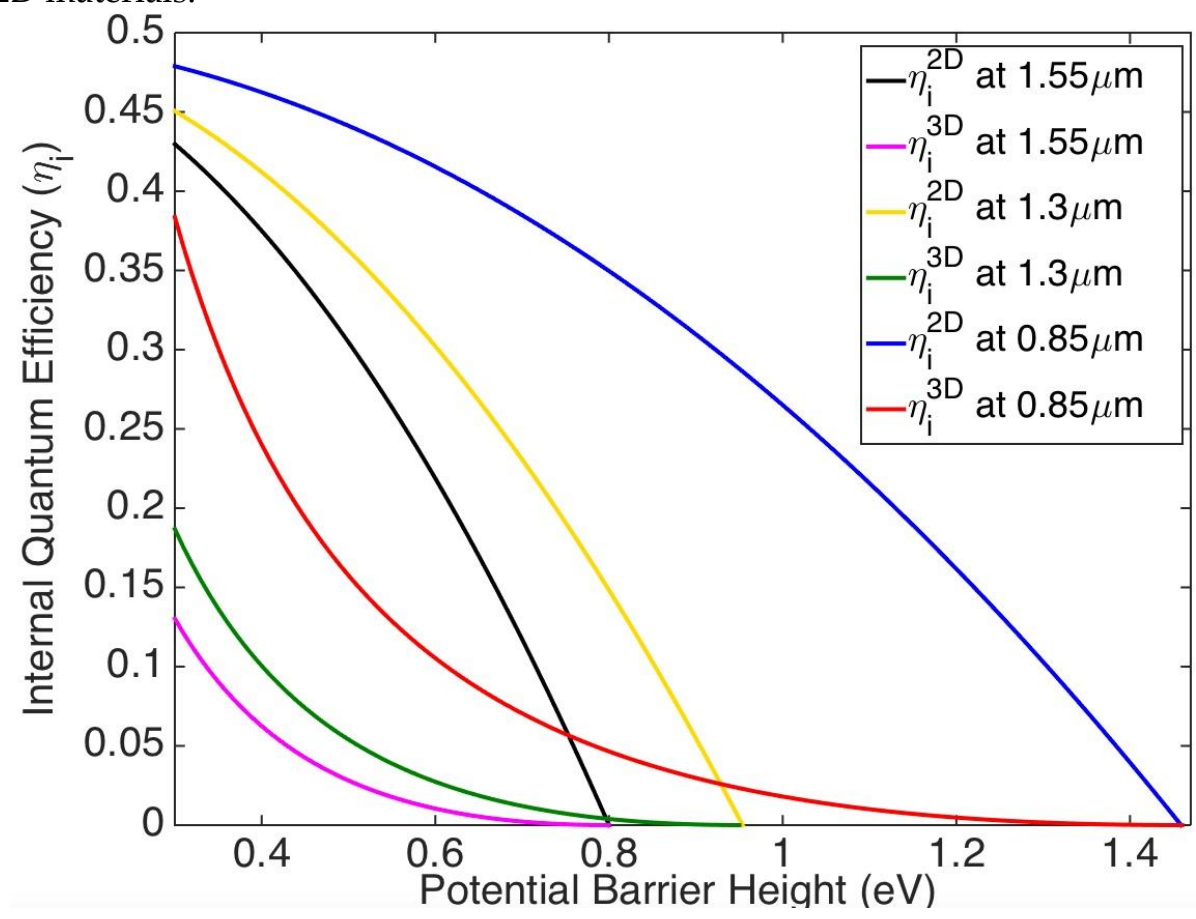


Figure 3. Comparison between internal quantum efficiency of 3D (Eq. 2) and 2D (Eq. 5) materials for three different wavelengths of $0.85,1.3$ and $1.55 \mu \mathrm{m}$.

\section{IPE-based silicon photodetectors based on graphene Schottky junctions}

Since its discovery in 2004, graphene has been deeply investigated [32]. Indeed, electrons move in graphene as massless particles making it suitable for fast electronics while the wide absorption (from the ultraviolet to the infrared) due to both intra-band and inter-band transitions [33,34] makes the material very useful in the photodetection field in particular for operation at NIR wavelengths. In the 2011, C.-C. Chen et al. have demonstrated that graphene is able to form a Schottky junction with Si [35] and this result opened the path to the investigation of IPE in the graphene/silicon junctions for NIR detection.

a)

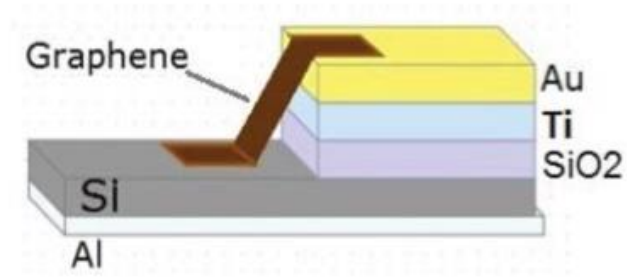

b)

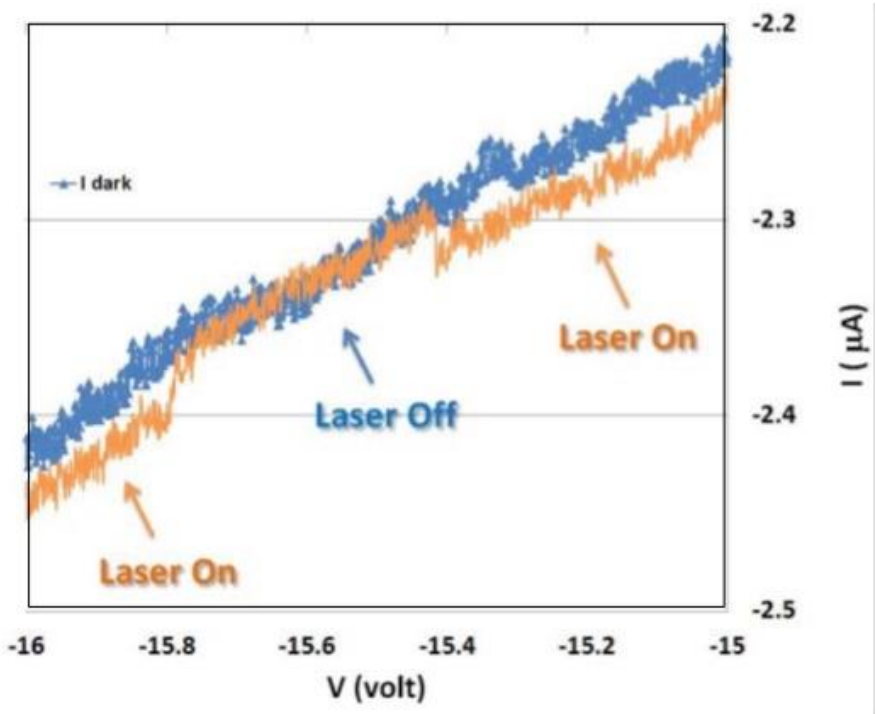

Figure 4. (a) Sketch describing the graphene-Si Schottky diode. (b) I-V curve at reverse bias (from -16 to $-15 \mathrm{~V}$ ). This figure demonstrates the change in diode current by switching on and off the laser at $1550 \mathrm{~nm}$.

NIR vertically illuminated IPE-based graphene/Si PDs were reported by M. Amirmazlaghani $e t$ al. in the 2013 [29]. These devices are based on exfoliated graphene and show a maximum experimental responsivity of $9.9 \mathrm{~mA} / \mathrm{W}$ at $1550 \mathrm{~nm}$ and $-16 \mathrm{~V}$ of reverse bias applied. Indeed, as shown in Fig. 4(b), the difference between the photogenerated and dark current is about 51.5nA under $5.2 \mu \mathrm{W}$.

In addition, a $2.4 \mu \mathrm{A}$ of dark current was measured while a SBH and ideality factor of 0.44-0.47 $\mathrm{eV}$ and 1.3-2.1 were extracted from the IV Schottky characteristic, respectively. In this paper the authors point out the experimental responsivity is higher than that one predicted by the traditional theory. This discrepancy has been explained by claiming that a new modified IPE theory should be derived if two-dimensional materials are involved in the junction. Indeed, they propose an alternative model (Eq. 5) resulting in a good agreement with the experiments. It is worth mentioning that very recently U. Levy at al. have proposed an alternative phenomenological model to explain physics behind the IPE enhancement in junctions involving 2D materials [36]. Subsequently, Goykhman et al. demonstrated a NIR graphene/silicon Schottky PD integrated with a waveguide realized starting from a silicon-on-insulator (SOI) substrate. In this device the single graphene layer (SLG) is grown by chemical vapour deposition CVD system [37] and the structure is shown in Figure 5 . 


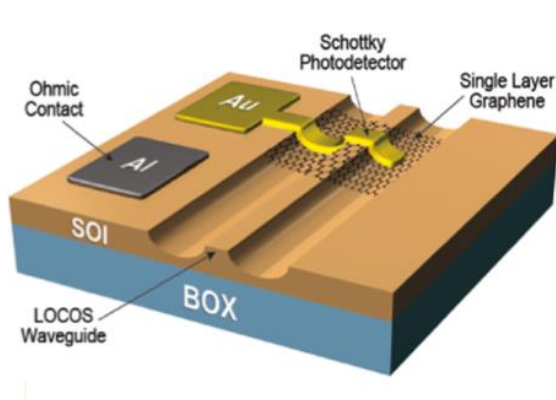

(a)

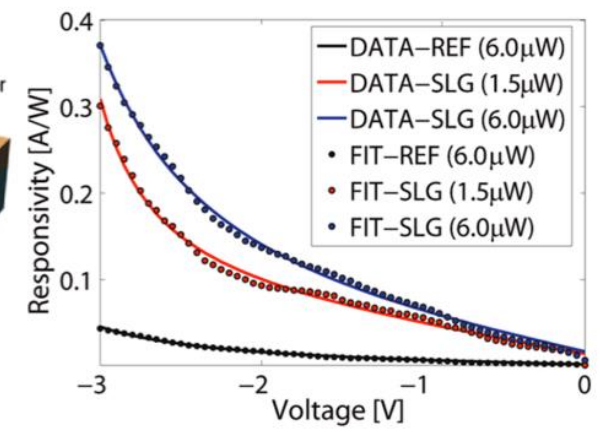

(b)

Figure 5. (a) Schematic Metal-SLG-Si Schottky PD proposed in [37]. (b) Responsivity of Metal-SLG-Si and reference Metal-Si PDs for a reverse bias ranging from 0 to $-3 \mathrm{~V}$ (reference Metal-Si device is used to demonstrate the key role of graphene). Solid lines show a fit of the bias dependent responsivity based on combined TFE and avalanche multiplication processes.

The PD is constituted by a Si rib waveguide on which has been deposited a SLG/Au layer acting as Schottky contact. The collecting Ohmic contact is realized in alluminum (Al) and deposited on the $\mathrm{Si}$ substrate as shown on Fig 7(a). The optical beam propagating along the $\mathrm{Si}$ waveguide is able to excite the plasmonic modes at SLG/Si interface. The PD length and width are $\sim 5 \mu \mathrm{m}$ and $310 \mathrm{~nm}$, respectively. At $1 \mathrm{~V}$ of reverse bias applied, a responsivity at $1550 \mathrm{~nm}$ and dark current of $0.085 \mathrm{~A} / \mathrm{W}$ and $20 \mathrm{nA}$ were reported, respectively. Finally, the authors show that responsivity abruptly increases up to $0.37 \mathrm{~A} / \mathrm{W}$ at $-3 \mathrm{~V}$. The authors explain this abruptly increase by the combined effect of two processes: avalanche multiplication within the Si depletion region and thermionic-field emission, TFE [38] through the graphene/Si Schottky junction. Under avalanche conditions, the dark current also increases up to $3 \mu \mathrm{m}$. The SBH extracted by the electrical measurement is $0.34 \mathrm{eV}$.

In the 2015, Vabbina et al. have reported on the fabrication and characterization of two-dimensional PD based on graphene/molybdene disulfide (MoS2) Schottky junction deposited on a SiO2/Si bulk substrate [39]. MoS2 has been deposited by a sputtering-CVD technique, while graphene by a CVD process. MoS2 is a 2D material having semiconductor characteristic with an indirect bandgap of 1.8 $\mathrm{eV}$. The graphene/MoS2 SBH is $0.139 \mathrm{eV}$ and at wavelengths higher than $670 \mathrm{~nm}$ the devices show a response that can be ascribed to IPE from the graphene to the MoS2. In particular, under an illumination of $1440 \mathrm{~nm}$ a NEP and a maximum responsivity of $7.8 \times 10-12 \mathrm{~W} / \sqrt{\mathrm{Hz}}$ amd $1.26 \mathrm{~A} / \mathrm{W}$ at $-2 \mathrm{~V}$, were reported. Dark current was measured as $300 \mu \mathrm{A}$ at $-2 \mathrm{~V}$. Finally the authors show that their device can also work in the visible spectrum taking advantage of the MoS2 linear absorption (energy gap excitation) reporting a maximum responsivity at $-2 \mathrm{~V}$ of $0.52 \mathrm{~A} / \mathrm{W}$ at $590 \mathrm{~nm}$. 


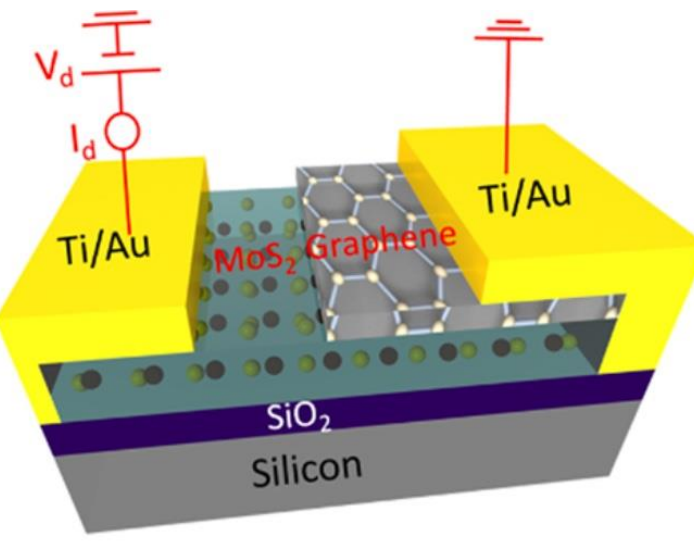

(a)

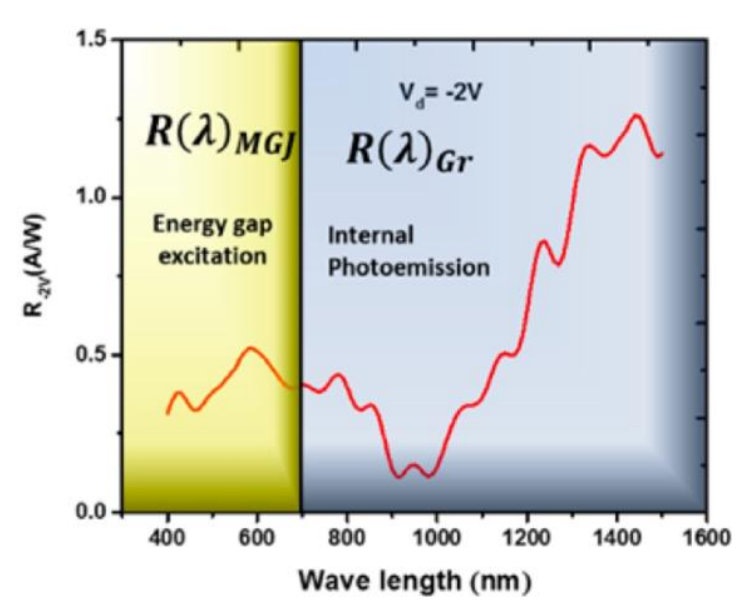

(b)

Figure 6. MoS2 /graphene Schottky PD described in Ref. [39]. (b) Device spectral response.

More recently, in 2017, Casalino et al. have reported on vertically-illuminated graphene/Si Schottky PDs incorporated into a Fabry-Perot optical microcavity [40]. The resonant cavity consists of a $\lambda / 2 \mathrm{Si}$ slab layer surrounded between graphene/Si top and Au bottom mirrors as shown in Fig.7a. The optical cavity is able to enhance the interaction of the light with graphene in order to increase its absorption. In this work the authors prove that the device responsivity peaks coincide with the resonances of the Fabry-Perot microcavity and that the responsivity increases with the number of light round trips of the cavity. Thanks to the multiple reflections in the cavity, the graphene absorption increases up to $8 \%$ leading to a maximum external (internal) responsivity increasing with the reverse voltage applied and reaching a maximum of $\sim 20 \mathrm{~mA} / \mathrm{W}(0.25 \mathrm{~A} / \mathrm{W})$ at $-10 \mathrm{~V}$ (Fig. 7(b)); this value is the highest reported so far for free-space illuminated Si PDs at $1550 \mathrm{~nm}$. In addition, the authors prove the SBH dependence on applied reverse voltage. Finally, the dark current at -10V have been measured as $147 \mu \mathrm{A}$ while the device bandwidth has been estimated in $120 \mathrm{MHz}$.

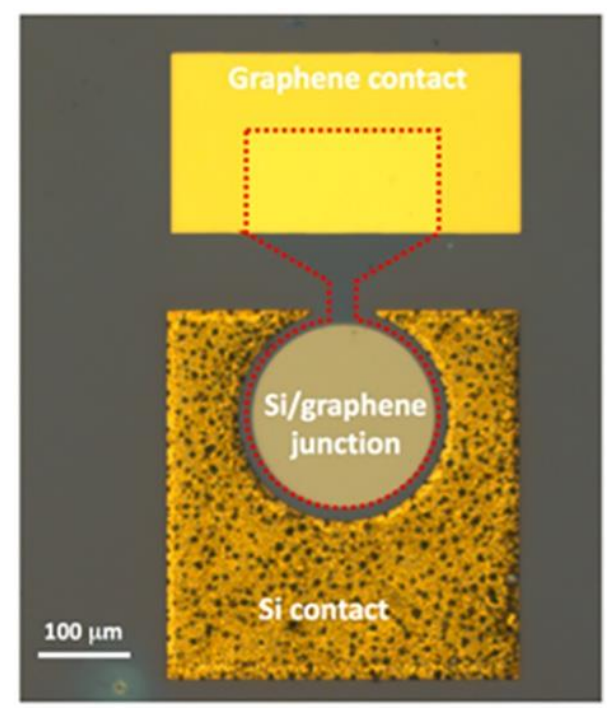

(a)

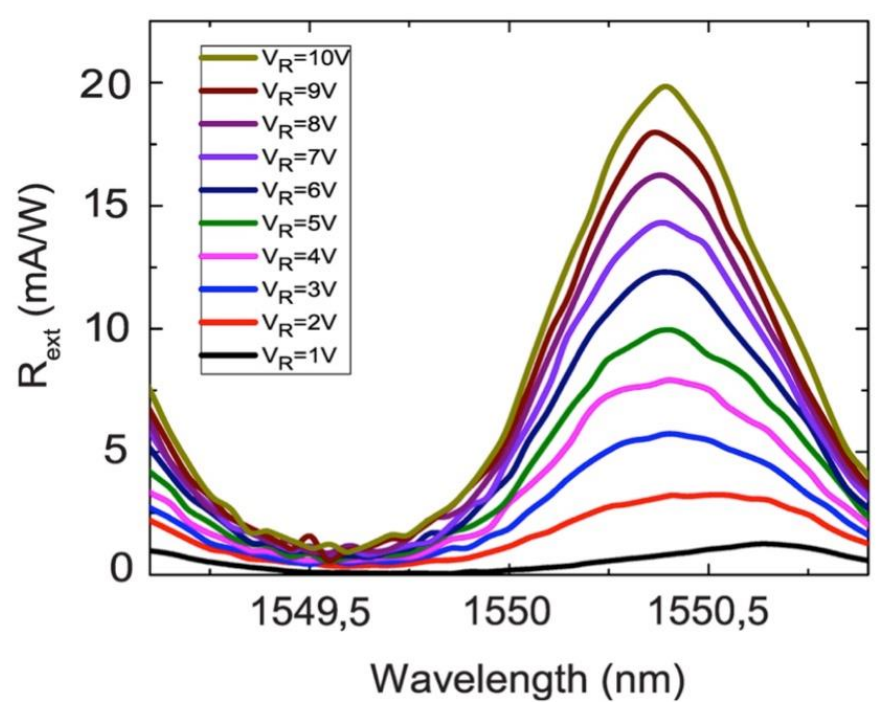

(b)

Figure 7. (a) Optical image of fabricated device investigated in Ref. 40. (b) External responsivity as a function of increased reverse voltage. 
In order to increase the low graphene absorption of the previous device (only 8\%), the same author has theoretically proposed a new concept of resonant cavity-enhanced (RCE) graphene/silicon Schottky PD working at $1550 \mathrm{~nm}$ [41]. Device is essentially a Fabry-Perot interferometer constituted by an optical cavity of hydrogenated amorphous silicon, graphene and crystalline silicon, surrounded by two high-reflectivity distributed Bragg reflectors as shown in Fig. 8(a). The optical field enhancement allows increasing the single-layer graphene optical absorption up to $100 \%$. The optoelectronic transduction mechanism is based on IPE through the crystalline silicon/graphene Schottky junction. In this work it has been theoretically proved that in an optimized device, an external quantum efficiency, and responsivity of $35 \%$, and $0.43 \mathrm{~A} / \mathrm{W}$ could be achieved, respectively, as shown in Fig. 8(b). Finally, device bandwidth and noise, in terms of dark current and normalized photocurrent-to-dark current ratio, have been discussed.

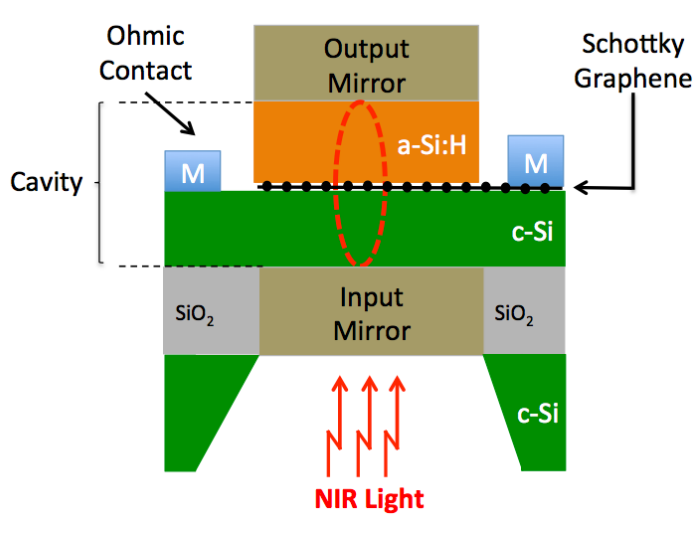

(a)
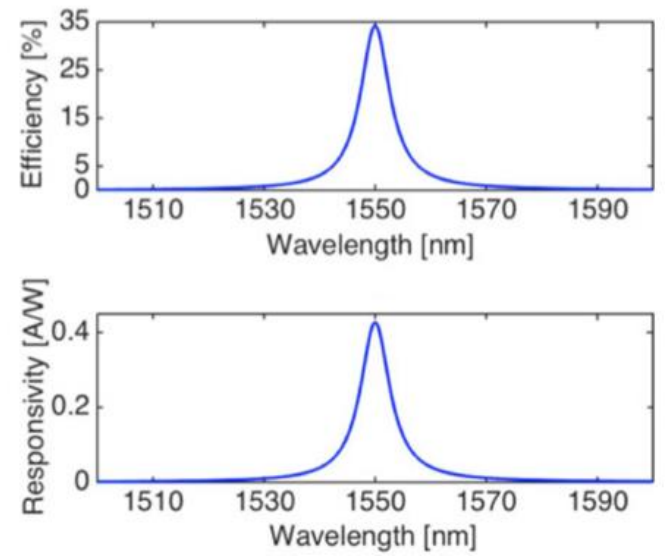

(b)

Figure 8. (a) Section sketch and (b) theoretical external quantum efficiency and responsivity of the high-finesse resonant cavity enhanced PD proposed in Ref. [41].

Very recently IPE through a graphene/silicon Schottky junction has demonstrated the capability to work also at wavelengths longer than $1550 \mathrm{~nm}$ [30]. Indeed, in Ref. 30 has been reported the first freespace Si-based PD able to work at $2 \mu \mathrm{m}$. The sketch of the proposed is shown in Fig. 9(a), they are based on graphene/Si Schottky junctions whose electrical parameters are carefully characterized at temperatures within the range from 280 to $315 \mathrm{~K}$. The Schottky barrier is $0.62 \mathrm{eV}$ at $300 \mathrm{~K}$ and shows a dependence on temperature ascribed to interface defects. Devices are characterized by an external responsivity of $0.16 \mathrm{~mA} / \mathrm{W}$ (Fig. 9(b)) under zero bias conditions corresponding to an internal maximum responsivities of at least $7.2 \mathrm{~mA} / \mathrm{W}$. Measured dark current is $\sim 3 \mu \mathrm{A}$ at $-6 \mathrm{~V}$ while the estimated bandwidth is in the $\mathrm{KHz}$ regime due to the very high series resistance. The proposed devices show the potentialities to work also at wavelength longer than 2 micron. A summary of all the aforementioned devices are reported in Table 1 for comparison. 


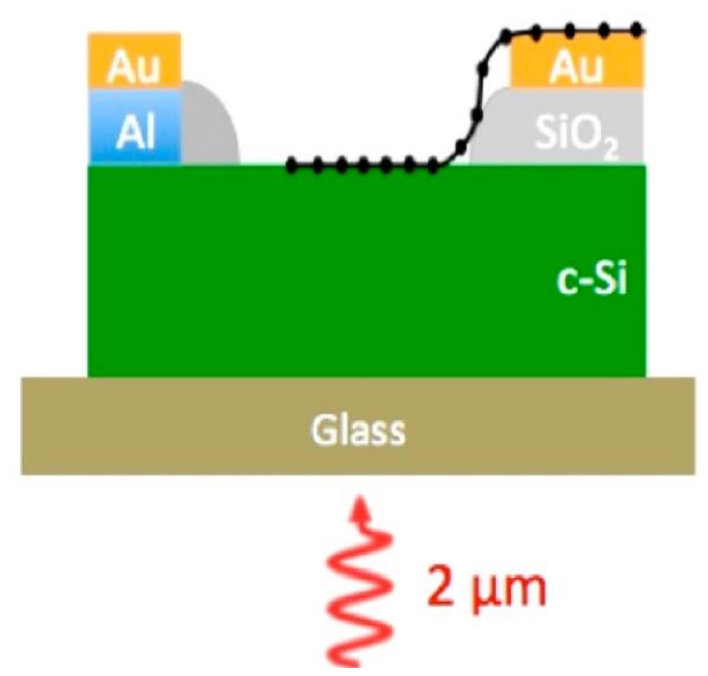

(a)

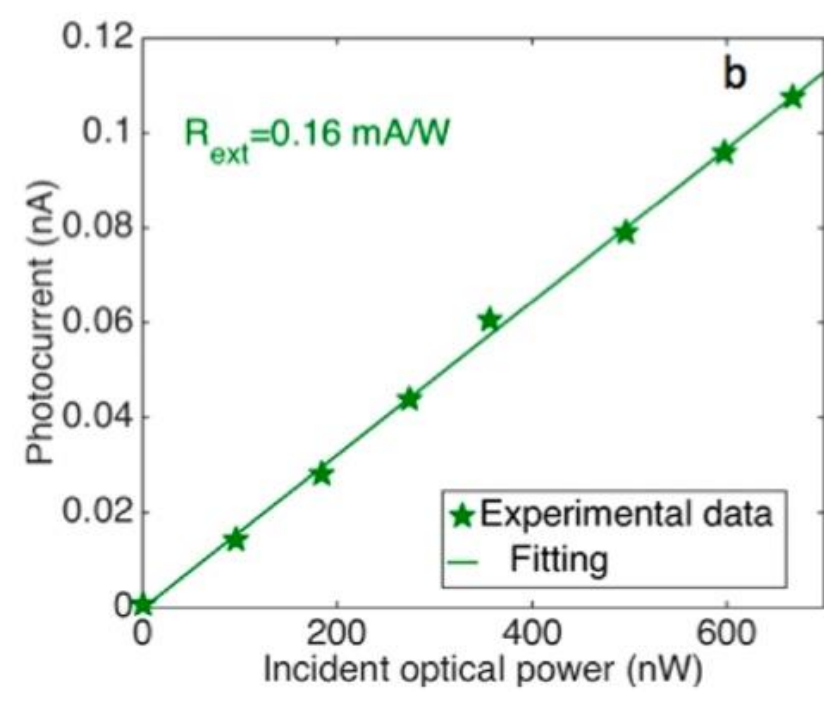

(b)

Figure 9. (a) Schematic cross-sectional view of the graphene/Si Schottky PD reported in Ref. 30, (b) Measured photocurrent vs incident optical power.

Table 1. Summary of selected near-infrared silicon Schottky photodetectors based on graphene.

\begin{tabular}{|c|c|c|c|c|c|c|}
\hline Ref. & $\begin{array}{c}\text { External } \\
\text { Responsivity }\end{array}$ & $\begin{array}{c}\text { Dark } \\
\text { current }\end{array}$ & Bandwidth & $\begin{array}{l}\text { SBH } \\
(\mathrm{eV})\end{array}$ & Type & Configuration \\
\hline [29] & $\begin{array}{c}9.9 \mathrm{~mA} / \mathrm{W} \text { at } 1550 \mathrm{~nm} \\
\text { and }-16 \mathrm{~V}\end{array}$ & $\sim 2.4 \mu \mathrm{A}$ at $-16 \mathrm{~V}$ & - & $0.44-0.46$ & Exfol. & Free-space \\
\hline$[37]$ & $\begin{array}{c}370 \mathrm{~mA} / \mathrm{W} \text { at } 1550 \mathrm{~nm} \\
\text { and }-3 \mathrm{~V}\end{array}$ & $\sim 3 \mu \mathrm{A}$ at $-3 \mathrm{~V}$ & - & 0.34 & CVD & Waveguide \\
\hline [39] & $\begin{array}{c}1.26 \mathrm{~A} / \mathrm{W} \text { at } 1440 \mathrm{~nm} \\
\text { and at }-2 \mathrm{~V}\end{array}$ & $\sim 300$ at $\mu \mathrm{A}$ at $-2 \mathrm{~V}$ & - & 0.136 & CVD & Free-space \\
\hline$[40]$ & $\begin{array}{c}20 \mathrm{~mA} / \mathrm{W} \text { at } 1550 \mathrm{~nm} \\
\text { and at }-10 \mathrm{~V}\end{array}$ & $\sim 147$ at $\mu \mathrm{A}$ at $-10 \mathrm{~V}$ & $\begin{array}{c}120 \mathrm{MHz} \\
\text { (Estimated) }\end{array}$ & 0.46 & CVD & Free-space \\
\hline$[41]^{*}$ & $\begin{array}{c}0.43 \mathrm{~A} / \mathrm{W} \text { at } 1550 \mathrm{~nm} \\
\text { and at } 0 \mathrm{~V}\end{array}$ & $\begin{array}{l}561 \mathrm{nA} \text { if graphene } \\
\text { radius }(\mathrm{gr})<15 \mu \mathrm{m}\end{array}$ & $\begin{array}{l}\text { GHz regime } \\
(\mathrm{gr}<15 \mu \mathrm{m})\end{array}$ & 0.45 & - & Free-space \\
\hline$[42]$ & $\begin{array}{c}0.16 \mathrm{~mA} / \mathrm{W} \text { at } 2000 \mathrm{~nm} \\
\text { and } 0 \mathrm{~V}\end{array}$ & $\sim 3 \mu \mathrm{A}$ at $-6 \mathrm{~V}$ & $\begin{array}{l}\text { KHz regime } \\
\text { (Estimated) }\end{array}$ & 0.62 & CVD & Free-space \\
\hline
\end{tabular}

*Theoretical work.

\section{Conclusions}

In this work an overview of near-infrared Si PDs based on IPE occurring in graphene, has been presented. Firstly, we have described as IPE theory needs to be properly modified in junctions involving two-dimensional material, showing as this effect is intrinsically enhanced with respect to classical device based on 3D materials (metals). Then we have described and discussed in details the most common configurations reported in recent literature including both vertically-illuminated and waveguide structures. Finally a quantitative comparison of selected device has been given and summarized in Table I.

In some cases, this new family of devices shows performance that are already comparable to the stateof-the-art Ge devices currently employed in Si photonics promising to play a key role in both telecom and datacom applications. In addition, these devices show the potentialities for operation at longer wavelengths in the region of $2 \mu \mathrm{m}$, opening new perspectives for new application including free- 
space optical communications, light-radars and optical coherence tomography. However the possibility to integrate 2D materials like graphene in Si technology for high-volume production remains a crucial point that needs to be properly addressed in the next future.

Funding: This work was funded from the European Union for ATTRACT under Grant Agreement no. 777222 and the Horizon 2020 Framework Programme for Research and Innovation (2014-2020).

Conflicts of Interest: The author declares no conflict of interest.

\section{References}

1. Yole Dèvelop., https://www.i-micronews.com/category-listing/product/silicon-photonics-2018.html

2. Koester, S.J.; Schaub, J.D.; Dehlinger, G.; Chu, J.O. TITLE. sLs:IEEE J. of Selected Topics in Quant. Electron. 2006, 12, 1489-1502. [icp:

3. Harame, D.L.; Koester, S.J.; Freeman, G.; Cottrel, P.; Rim, K.; Dehlinger, G.; Ahlgren, D.; Dunn, J.S.; Greenberg, D.; Joseph, A.; Anderson, F.; Rieh, J.-S.; Onge, S. A. S. T.; Coolbaugh, D.; Ramachandran, V.; Cressler, J.D.; Subbanna, S. TITLE. Appl. Surf. Sci. 2004, 224, 9-17. is:p?

4. Koester, S. J.; Schaub, J. D.; Dehlinger, G.; Chu, J. O. Germanium-on-SOI infrared detectors for integrated photonic applications. IEEE J. Sel. Top. Quantum Electron. 2006, 12 (6), 1489-1502.

5. Harame, D.; Koester, S.; Freeman, G.; Cottrel, P.; Rim, K.; Dehlinger, G.; Ahlgren, D.; Dunn, J.; Greenberg, D.; Joseph, A. The revolution in SiGe: impact on device electronics. Appl. Surf. Sci. 2004, 224 (1), 9-17.

6. Wang, J.; Lee, S. Ge-Photodetectors for Si-Based Optoelectronic Integration. Sensors 2011, 11, 696-718.

7. Casalino, M. Internal photoemission theory: comments and theoretical limitations on the performance of near-infrared silicon schottky photodetectors. IEEE J. Quantum Electron. 2016, 52 (4), 1- 10.

8. Scales, C.; Berini, P. Thin-Film Schottky Barrier Photodetector Models. IEEE J. of Quant. Electron. 2010, 46, 633-643.

9. Elabd, H.; Villani, T.; Kosonocky, W.F. Palladium-Silicide Schottky-Barrier IR-CCD for SWIR Applications at Intermediate Temperatures. IEEE-ED Letters 1982, EDL-3, 89.

10. Elabd, H.; Villani, T.S.; Tower, J.R. High Density Schottky-Barrier IRCCD Sensors for SWIR Applications at Intermediated Temperature. SPIE's Tech. Symp. East 1982, Arlington (VA).

11. Kosonocky, W.F.; Elabd, H.; Erhardt, H.G.; Shallcross, F.V.; Villani, T.; Meray, G.; Cantella, M.J.; Klein, J.; Roberts, N. 64 x 128-elements High-Performance PtSi IR-CCD Image Sensor. IEDM 1981, Washington DC.

12. Kosonocky, W. F.; Elabd, H.; Erhardt, H.G.; Shallcross, F.V.; Meray, G.M.; Villani, T.S.; Groppe, J.V.; Miller, R.; Frantz, V.L.; Cantella, M.J.; Klein, J.; Roberts, N. Design and Performance of 64 x 128-Element PtSi Schottky-Barrier IR-CCD Focal Plane Array. SPIE's Tech. Symp. East 1982, Arlington (VA).

13. Kosonocky, W.F.; Elabd, H.; Erhardt, H.G.; Shallcross, F.V.; Meray, G.M.; Miller, R.; Villani, T.; Groppe, J.V.; Frantz, V.L.; Tams III, F.J. Schottky-Barrier Infrared Image Sensors. 27 May-3 June 1982, 49-58.

14. Casalino, M.; Sirleto, L.; Moretti, L.; Della Corte, F.; Rendina, I. Design of a silicon resonant cavity enhanced photodetector based on the internal photoemission effect at $1.55 \mu \mathrm{m}$. Journal of Optics A: Pure and applied optics 2006, 8, 909-913.

15. Casalino, M.; Sirleto, L.; Moretti, L.; Gioffrè, M.; Coppola, G.; Rendina, I. Silicon resonant cavity enhanced photodetector based on the internal photoemission effect at 1.55 micron: Fabrication and characterization. Appl. Phys. Lett. 2008, 92, 251104. 
16. Zhu, S.; Chu, H.S.; Lo, G.Q.; Bai, P.; Kwong, D.L. TITLE. Applied Physics Letters 2012, 100, 061109.

17. Berini, P.; Olivieri, A.; Chen, C. TITLE. Nanotechnology 2012, 23, 444011.

18. Akbari, A.; Tait, R.N.; Berini, P. TITLE. Optics Express 2010, 18, 8505-8514.

19. Sobhani, A.; Knight, M.W.; Wang, Y.; Zheng, B.; King, N.S.; Brown, L.V.; Fang, Z.; Nordlander, P.; Halas, N.J. Nature Communications 2013, 4(1643), 1-6.

20. Knight, M.W.; Sobhani, H.; Nordlander, P.; Halas, N.J. TITLE. Science 2011, 332, 702-704.

21. Desiatov, B.; Goykhman, I.; Mazurski, N.; Shappir, J.; Khurgin, J.B.; Levy, U. Plasmonic enhanced silicon pyramids for internal photoemission Schottky detectors in the near-infrared regime. Optica 2015, 2, 335-338.

22. Elabd, H.; Kosonocky, W.F. Theory and measurements of photoresponse of thin film Pd2Si and PtSi Schottky-barrier detectors with optical cavity. RCA Review 1982, 43, 569.

23. Vickers, V. E. Model of Schottky barrier hot-electron-mode photodetection. Appl. Opt. 1971, 10, 2190.

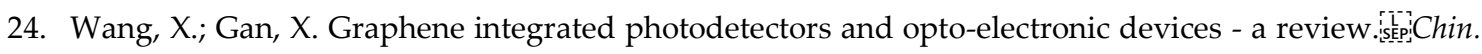
Phys. B 2017, 26(3), 034201.

25. Koppens, F.H.L.; Mueller, T.; Avouris, Ph.; Ferrari, A.C.; Vitiello, M.S.; Polini, M. Photodetectors based on graphene, other two-dimensional materials and hybrid systems. Nature Nanotechnology 2014, 9, 780793.

26. Lee, E.J.; Balasubramanian, K.; Weitz, R.T.; Burghard, M.; Kern, K. Contact and edge effects in graphene devices. Nature Nanotechnol. 2008, 3, 486-490.

27. Fowler R.H. The analysis of photoelectric sensitivity curves for clean metals at various temperatures. Phys. Rev. 1931, 38, 45-56.

28. Cohen, J; Vilms, J; Archer, R.J. Investigation of Semiconductor Schottky Barriers for Optical Detection and Cathodic Emission. Air Force Cambridge Research Labs. 1968; Report No. 68-0651.

29. Amirmazlaghani, M.; Raissi, F.; Habibpour, O.; Vukusic, J.; Stake, J. Graphene-Si Schottky IR detector. IEEE J. Quant. Elect. 2013, 49, 2589.

30. Casalino, M.; Russo, R.; Russo, C.; Ciajolo, A.; Di Gennaro, E.; Iodice, M. Free-Space Schottky Graphene/Silicon Photodetectors operating at $2 \mu \mathrm{m}$. ACS Photonics 2018, 5(11), 4577-4585.

31. Van Tuan, D. Electronic and Transport Properties of Graphene. In Charge and Spin Transport in Disordered Graphene-Based Materials; Publisher: Springer, Cham, 2016, pp. 10.

32. Novoselov, K.S.; Fal'ko, V.I.; Colombo, L.; Gellert, P.R.; Schwab, M.G.; Kim, K. A roadmap for graphene. Nature 2012, 490, 192-200.

33. Dawlaty, J.; Shivaraman, S.; Strait, J.; George, P.; Chandrashekhar, M.; Rana, F.; Spencer, M.G.; Veksler, D.; Chen, Y. Measurement of the optical absorption spectra of epitaxial graphene from terahertz to visible. Appl. Phys. Lett. 2008, 93, 131905-1-13.

34. Sensale-Rodriguez, B; Yan, R.; Kelly, M.M.; Fang, T.; Tahy, K.; Hwang, W.S.; Jena, D.; Liu, L.; Xing, H.G. Efficient terahertz electro-absorption modulation employing graphene plasmonic structures. Appl. Phys. Lett. 2012, 101, 261115.

35. Chen, C.-C.; Aykol, M.; Chang, C.-C.; Levi, A.F.J.; Cronin, S.B. Graphene-silicon Schottky diodes. Nano Letters 2011, 11, 1863-1867.

36. Levy, U.; Grajower, M.; Goncalves, P.A.D.; Mortensen, N.A.; Khurgin, J.B. Plasmonic silicon Schottky photodetectors: The physics behind graphene enhanced internal photoemission. APL Photonics 2017, 2 , 026103. 
37. Goykhman, I.; Sassi, U.; Desiatov, B.; Mazurski, N.; Milana, S.; De Fazio, D.; Eiden, A.; Khurgin, J.; Shappir, J.; Levy, U.; Ferrari, A.C. On-Chip Integrated, Silicon-Graphene Plasmonic Schottky Photodetector with High Responsivity and Avalanche Photogain. Nano Lett. 2016, 16, 3005-3013.

38. Padovani, F.A.; Stratton, R. Field and thermionic-field emission in Schottky barriers. Solid State Electron. 1966, 9, 695-707.

39. Vabbina, P.; Choudhary, N.; Chowdhury, A.-A.; Sinha, R.; Karabiyik, M.; Das, S.; Choi, W.; Palaoi, N.; Pala, N. Highly Sensitive Wide Bandwidth Photodetector Based on Internal Photoemission in CVD Grown p-Type MoS2/Graphene Schottky Junction. ACS Appl. Mater. Interfaces 2015, 7, 15206-15213.

40. Casalino, M; Sassi, U.; Goykhman, I.; Eiden, A.; Lidorikis, E.; Milana, S.; De Fazio, D.; Tomarchio, F.; Iodice, M.; Coppola, G.; Ferrari, A. C. Vertically Illuminated, Resonant Cavity Enhanced, GrapheneSilicon Schottky Photodetectors. ACS Nano 2017, 11, 10955-10963.

41. Casalino, M. Design of Resonant Cavity-Enhanced Schottky Graphene/Silicon Photodetectors at 1550 nm. Journal of Ligthwave Technology 2018, 36 1766-1774. 